\title{
CONTROLS OF DEPOSITIONAL ENVIRONMENTS ON RESERVOIR QUALITY IN TERMS OF POROSITY AND PERMEABILITY GABO FIELD NIGER DELTA.
}

\author{
Nduaguibe, T. W and Ideozu, R. U. \\ Department of Geology, University of Port Harcourt, Rivers State, Nigeria. \\ Corresponding author email: richmond.ideozu@uniport.edu.ng
}

Abstract

The controls of depositional environments on reservoir quality have been evaluated in terms of porosity and permeability of the Gabo Field, Niger Delta, Nigeria. Data used in this research include Well logs, Core data and photos, and grain size analysis for Wells 51 and 52 in the study area. Standard methods as applicable in petrophysical and sedimentological analysis has been adopted. Thirteen reservoir units have been identified in wells 51 and 52 which had 5 reservoirs cored each. The lithofacies units of the identified reservoirs across the study area, comprise pebbly sands, coarse -, medium -, fine- and very fine-grained sands, sandy mud, silty sands and heteroliths. The heteroliths - very fine-grained silty muds are highly bioturbated. Ophiomorpha and skolithos are the major trace fossils with sedimentary structures (ripple lamination, wavy lenticular and planar beds, cross bedded sands, coarsening and fining upward). The facies associations interpreted for the study area are Channel and Coastal barrier systems and the environment of deposition as distributary channel, upper and lower shoreface. The sedimentary processes that deposited facies ranged from high energy regimes, reworking by waves to low energy with periodic influx of silts and muds. The average porosity and permeability for reservoirs in Well 51 is $16.7 \%$ and $1317 \mathrm{Md}$, reservoirs in Well 52 is $28.2 \%$ and 2330Md whereas porosity range for the study area is $2 \%$ - 32\% and permeability is $1.2-10600 \mathrm{Md}$. The reservoir quality reservoir of the sand units in Well 51 (7, 9 and 13) and Well $52(5,7,9,11$ and 13) is excellent - good, this is because of the dynamics environments of deposition (upper shoreface and distributary channel) as well as the mechanisms that play out during deposition such as bioturbation, sorting, sedimentary structures formed. Whereas the poor quality across the reservoirs especially the lower shoreface and prodelta facies is as result of lack bioturbation, connectivity, multiplicity of burrows that may have been plugged by clay and intercalation of shale and sand (heteroliths). This research has shown that environments of deposition have direct influence the reservoir quality in terms of porosity and permeability.

Key words: Porosity, permeability, skolithos, Ophiomorpha, lithofacies, reservoir, bioturbation, channel and coastal barrier systems.

\section{Introduction}

The Gabo Field is one of the oil fields in the Niger Delta Basin. Most Wells drilled in the Tertiary Niger Delta have penetrated several sandstone units in the Benin and Agbada Formations, separated by clay/shale brakes (Reyment, 1965). The sandstone units' thickness apparently increases upward while the shale thickness appears to be increasing downward and probably overlies a Basement Complex, (Short and Stauble, 1967). Niger Delta covers an area of 75,000 square kilometers, opens to the South Atlantic Ocean and out builds into the Gulf of Guinea which is probably an extension of the Benue Trough. It merges westward across Okitipupa and Dahomey Embayment, (Reyment, 1967). The quality of a reservoir sand unit in a depositional system can be related to its: porosity, permeability, type of depositional environment, thickness and its lateral continuity, these in turn are influenced by the textural features such as sorting, grain size, roundness, sphericity, cement, clay content, the presence of trace fossils, sedimentary process and structures and energy of deposition of medium. This research seeks to evaluate the influence of depositional environment and its mechanisms on reservoir quality of Gabo Field in relation to porosity and permeability and how it can contribute to the optimization of resource exploration and exploitation within the Gabo Field. The study area is located within the south-western part of the coastal swamp Depobelt region of Niger Delta (Figure 1-2). The geology of the Niger Delta has been well established in addition to the stratigraphy, structural framework and petroleum geology (Doust and Omatsola, 1989, 1990; Reijers, 1996; Kulke, 1995; Ekweozor and Daukoru, 1994; Evamy et al, 1978). See Figure 3-4. 


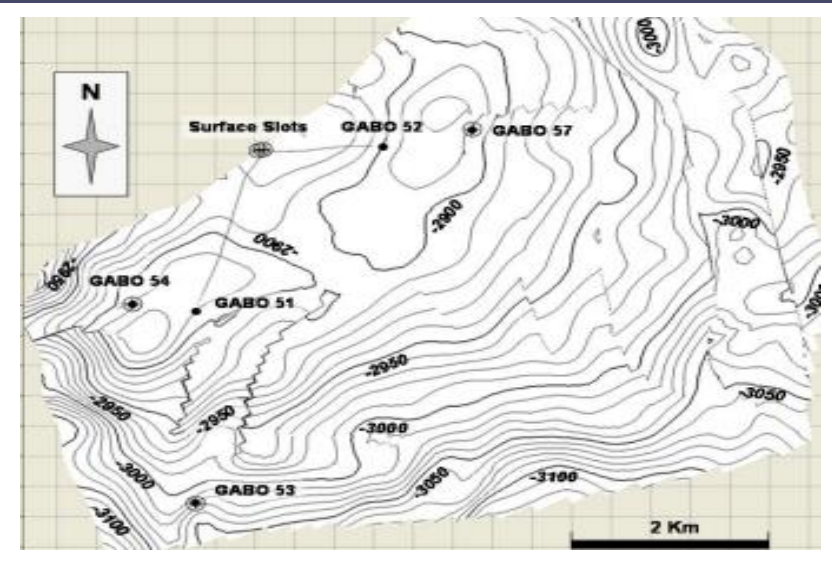

Figure 1 Base map of Gabo Filed

Source Total E and P 2014

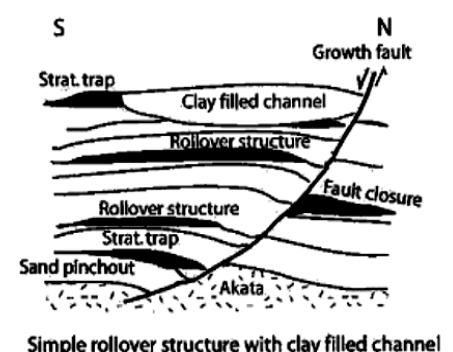

$\mathrm{S}$

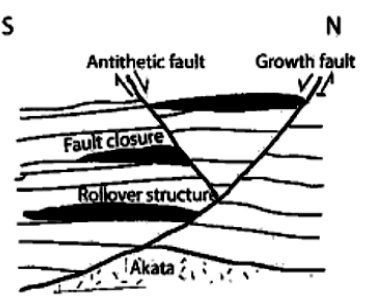

Structure with antithetic fault

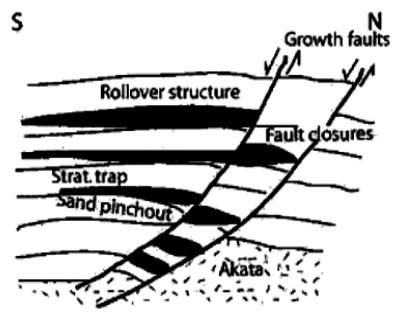

Structure with multiple growth faults

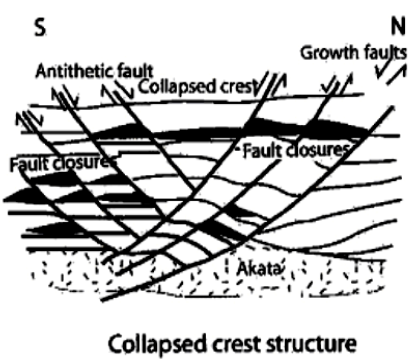

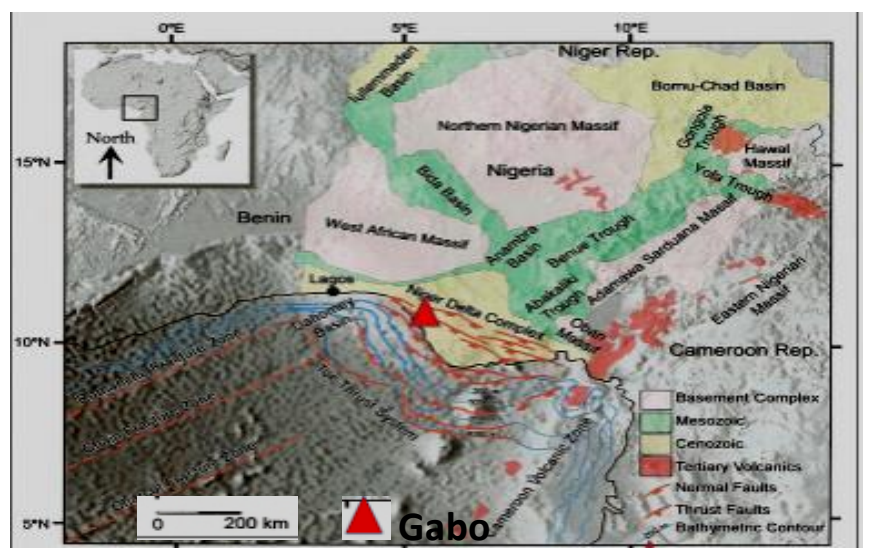

Figure 2 Location of Gabo Filed

(modified from Mitchum, 2006)
Figure 3 Niger trapping systems

(Modified from Doust and Omatsola (1990)

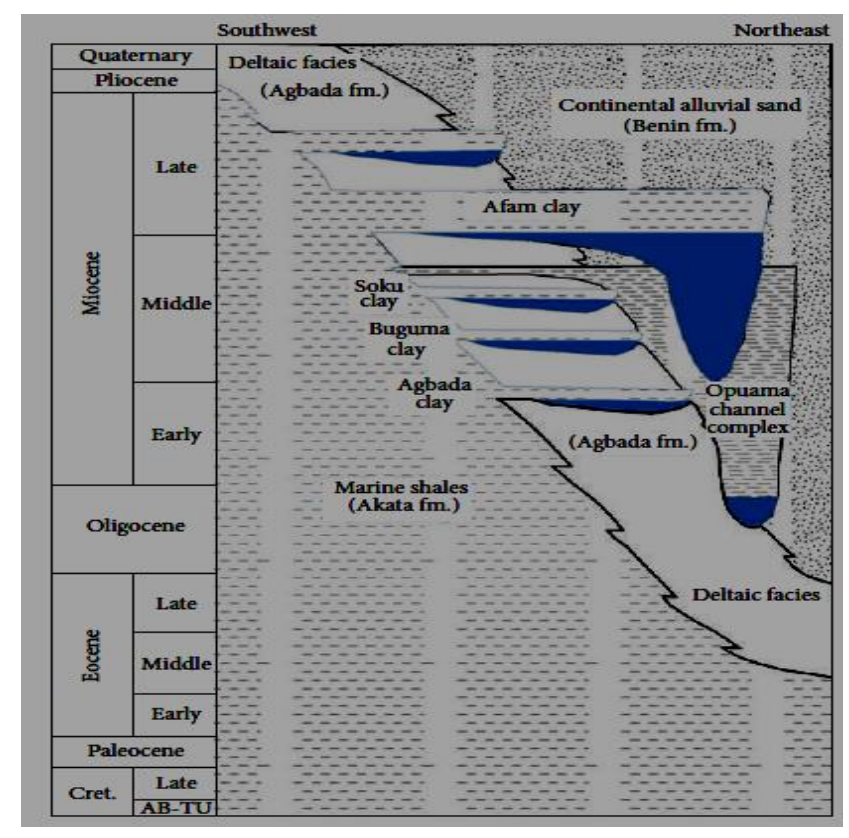

Figure 4 Stratigraphic structure of the Niger Delta (After Shanon and Naylor 1989; Doust and Omatshola (1990)

and Stacher (1995).

\section{Materials and Methods}

\section{Materials.}

The materials of this research have been provided by an International Oil Company in Nigeria (for propriety reasons, the name of the company and field is omitted) through the Department of Petroleum Resources (DPR). The data set provided include the following:

1. Well logs and mud logs

2. Core photos and core data

3. Lithofacies description

4. Location map

\section{Methods}

The work flow diagram illustrates the methodology applied in this research (Figure 5). Quantitative petrophysical analysis and evaluation was carried out on the two wells to determine their Porosity $(\phi)$ and Permeability $(\mathrm{K})$ from 
the well $\log$ s compared with core data. See Figure 3. The formula upon which the software computes the petrophysical parameters are shown below.

\section{Effective Porosity}

$$
\phi_{\text {eff }}=\phi_{D}-\left(\mathbf{V}_{\text {sh }} \times \phi_{D \text { Dh }}\right)
$$

Where:

$\phi_{\text {eff }}=$ Effective porosity

$\phi_{\mathrm{D}}=$ Total porosity

$\mathrm{V}_{\text {sh }}=$ Shale volume

$\phi_{\text {Dsh }}=$ Shale porosity from density log

$\left.\mathbf{G R}_{\mathbf{i}}=\left(\mathbf{G R}_{\log }-\mathbf{G R} \mathbf{R}_{\min }\right)-\mathbf{G} \mathbf{R}_{\min }\right) /\left(\mathbf{G R} \mathbf{R}_{\max }-\mathbf{G R}_{\min }\right)$

$\mathrm{V}_{\text {sh }}=0.083 \times\left(2^{(3.7 \times \text { GRi })}-1\right)$

Where: GRi $=$ Gamma ray index,

$\mathrm{GR}_{\log }=$ Gamma ray log reading,

$\mathrm{GR}_{\min }=$ Minimum Gamma ray log reading, which signifies clean sand and $\mathrm{GR}_{\max }=$ Maximum Gamma ray log reading, which signifies $100 \%$ shale. Both equations calculate the volume of shale.

\section{Permeability}

$$
\mathbf{K}=\left(250 \times \phi_{\text {eff }}{ }^{3} / S_{\text {wirr }}\right)^{2} \text { [Tixer equation] }
$$

Where:

$\mathrm{K}=$ Permeability

$\phi_{\text {eff }}=$ Effective porosity

$\mathrm{S}_{\text {wirr }}=$ Irreducible Water Saturation

\section{Results and Discussion}

The results of this research are presented in Figures 6 - 8 and Tables 1 - 15.In Well 51, the total cored intervals are $3687-3719 \mathrm{~m}(32 \mathrm{~m}), 3764-3794(30 \mathrm{~m})$ and $34078-4129(51 \mathrm{~m})$. Three reservoir units have been delineated 7 ( $a$ and b), 9 ( $a$ and $b$ ) and 13 ( $a$ and b). See Tables 1 - 5. In Well 52, the total cored intervals are $3687-3719 \mathrm{~m}(32$ $\mathrm{m}), 3764-3794(30 \mathrm{~m})$ and $34078-4129(51 \mathrm{~m})$. Three reservoir units have been delineated 7 (a and b), 9 (a and b) and 13 ( $\mathrm{a}$ and $\mathrm{b})$. See Tables 6 - 10 .

\section{Discussion}

\section{Environment of Deposition (EoD)}

Environments of deposition (EoD) play a key role in reservoir characterization as well as in reservoir quality and performance predictions across a field (Toba and Ideozu, 2017, 2018). Reservoir sand bodies deposited in different depositional environments are characterized by different sand shape and geometry, size and heterogeneity. The depositional environment of the reservoirs have been interpreted based on well logs motifs using standard shape of GR-log (Figures 6 -7) and interpretation from core photos. Clastic sedimentary facies display characteristic vertical profiles based on which grain size, fining upward sequence or coarsening upward sequences. Determination of such these vertical variations in grain size from GR-log is valuable in the interpretation of depositional environments. See Figures 6 -7. In Well 51 the environments of deposition comprise fluvial channels, distributary channels, upper shoreface, lower shoreface and prodelta (See Tables 2 -5) whereas in Well 52 the environments of deposition comprise flood plain, fluvial channels, distributary channels, upper shoreface, lower shoreface and prodelta. See Tables $6-10$. 


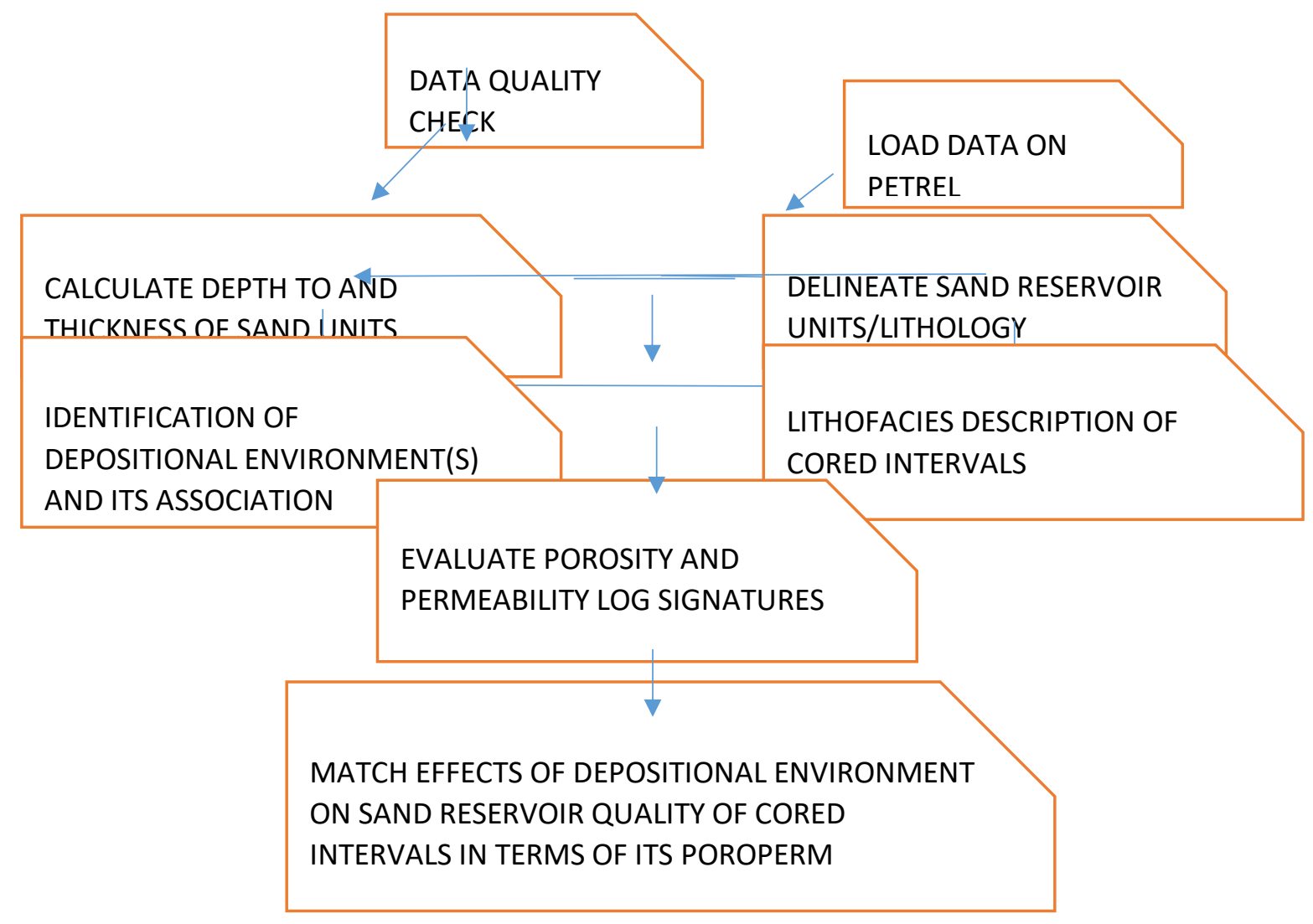

Figure 5 Work-flow chart

Table 1 Reservoir thickness in well 51.

\begin{tabular}{|l|l|l|l|l|l|l|}
\hline Reservoir & Lithology & Top $(\mathbf{m})$ & $\begin{array}{l}\text { Bottom } \\
(\mathbf{m})\end{array}$ & $\begin{array}{l}\text { Thickness } \\
(\mathbf{m})\end{array}$ & $\begin{array}{l}\text { Number of } \\
\text { cores }\end{array}$ & $\begin{array}{l}\text { Cored } \\
\text { length m }\end{array}$ \\
\hline $\mathbf{7}$ & Sand & 3686 & 3699 & 13 & 1 & 30 \\
\hline $\mathbf{9}$ & Sand & 3773 & 3791.5 & 18.5 & 1 & 30 \\
\hline $\mathbf{1 1}$ & Sand & 4087 & 4109.40 & 22.4 & 2 & 46.3 \\
\hline $\mathbf{1 3}$ & Sand & 4097.3 & 4098 & 0.7 & Un cored & - \\
\hline
\end{tabular}


Table 6 Reservoir thickness in well 52.

\begin{tabular}{|l|l|l|l|l|l|l|}
\hline Reservoir & Lithology & Top $(\mathbf{m})$ & $\begin{array}{l}\text { Bottom } \\
(\mathbf{m})\end{array}$ & $\begin{array}{l}\text { Thickness } \\
(\mathbf{m})\end{array}$ & $\begin{array}{l}\text { Number } \\
\text { of cores }\end{array}$ & $\begin{array}{l}\text { Cored } \\
\text { length } \mathbf{~ m}\end{array}$ \\
\hline $\mathbf{5}$ & Sand & 3324 & 3336 & 12 & 1 & 17 \\
\hline $\mathbf{7}$ & Sand & 3445 & 3468 & 23 & 1 & 30 \\
\hline $\mathbf{9}$ & Sand & 3542 & 3556 & 14 & 1 & 18 \\
\hline $\mathbf{1 1}$ & Sand & 3690.5 & 3698 & 7.5 & 1 & 18 \\
\hline $\mathbf{1 3}$ & Sand & 3797 & 3810 & 13 & 1 & 17 \\
\hline
\end{tabular}

\section{Reservoir Quality}

The quality of a reservoir rock can be evaluated in terms of porosity and permeability. Hydrocarbon reservoir sands that are thick enough, highly porous and permeable give better prospects, higher volume and profit and as such, major Oil Companies indicate keen interest in such reservoir sands. The reservoir quality of the sand units studied (Well 51: sands 7, 9 and 13; Well 52: sands 5, 7, 9,11 and 13) have higher quality because of their environments of deposition as well as the mechanisms that play out in them such as bioturbation, textures and sedimentary structures interpreted as distributary channels, fluvial channels and upper shoreface (See Tables $2-3$ and $6-7$ ). The least quality reservoirs occur probably due to lack of bioturbation, connectivity and type of lithology interpreted as flood plain, lower shoreface (heteroliths) and prodelta shales. See Tables $4-5$ and $8-9$. 
Table 2 Core Description, Deposition Environment, Porosity and Permeability for reservoir units in Well 51

\begin{tabular}{|c|c|c|c|c|c|c|c|}
\hline Reservoir & $\begin{array}{l}\text { Reservoir } \\
\text { unit }\end{array}$ & $\begin{array}{l}\text { Depth } \\
\text { range }(\mathrm{m})\end{array}$ & $\begin{array}{l}\text { Thicknes } \\
\text { s (m) }\end{array}$ & $\begin{array}{l}\text { Lithologic description / } \\
\text { sedimentary processes }\end{array}$ & $\begin{array}{l}\text { Environment } \\
\text { of } \\
\text { Deposition }\end{array}$ & $\begin{array}{l}\text { Porosity } \\
\text { Mean and } \\
\text { Range (\%) }\end{array}$ & $\begin{array}{l}\text { Permeability } \\
\text { Mean and range } \\
\text { (md) }\end{array}$ \\
\hline \multirow[t]{2}{*}{7} & $\mathrm{a}$ & $\begin{array}{l}3687- \\
3697\end{array}$ & 10 & $\begin{array}{l}\text { Medium to coarse grained, cross } \\
\text { bedded sandstone, poorly sorted, } \\
\text { low angle crossbedding, } \\
\text { bioturbated- ophiomorpha. High } \\
\text { energy flow regime }\end{array}$ & $\begin{array}{l}\text { Fluvial } \\
\text { channel }\end{array}$ & $\begin{array}{l}21.5 \\
16-28\end{array}$ & $\begin{array}{l}1047 \\
600-1600\end{array}$ \\
\hline & $\mathrm{b}$ & $\begin{array}{l}3710- \\
3718\end{array}$ & 8 & $\begin{array}{l}\text { Coarsening upward very fine to } \\
\text { fine- grained cross ripple } \\
\text { laminated sandstone, low angle } \\
\text { crossbedding to current ripple } \\
\text { bedding. High energy regime } \\
\text { reworked by waves }\end{array}$ & Upper shoreface & $\begin{array}{l}25.25 \\
16-29\end{array}$ & $\begin{array}{l}1037.5 \\
500-1400\end{array}$ \\
\hline \multirow[t]{2}{*}{9} & $\mathrm{a}$ & $\begin{array}{l}3773- \\
3783\end{array}$ & 10 & $\begin{array}{l}\text { Pebbly-coarse medium grained } \\
\text { cross bedded fining upward } \\
\text { sandstone, planar cross bedded to } \\
\text { low angle crossbedding, poorly } \\
\text { sorted with bioturbation. Trace } \\
\text { fossil present include } \\
\text { ophiomorpha and skolithos. High } \\
\text { energy flow regime. }\end{array}$ & $\begin{array}{l}\text { Fluvial } \\
\text { Channel }\end{array}$ & $\begin{array}{l}21.89 \\
8-29\end{array}$ & $\begin{array}{l}1210.07 \\
500-1800\end{array}$ \\
\hline & $\mathrm{b}$ & $\begin{array}{l}3783- \\
3790\end{array}$ & 7 & $\begin{array}{l}\text { Fine to very fine-grained well } \\
\text { sorted sandstone, wave rippled to } \\
\text { current bedding and planar } \\
\text { bedding. High energy regime } \\
\text { reworked by waves }\end{array}$ & Upper shoreface & $\begin{array}{l}22.2 \\
4-29\end{array}$ & $\begin{array}{l}780 \\
6-1800\end{array}$ \\
\hline \multirow[t]{2}{*}{13} & $\mathrm{a}$ & $\begin{array}{l}4088- \\
4101\end{array}$ & 12 & $\begin{array}{l}\text { Medium to coarse-grained cross } \\
\text { bedded blocky sandstone, low } \\
\text { angle crossbedding moderate to } \\
\text { poorly sorted, bioturbated, present } \\
\text { is ophiomorpha and planolites. } \\
\text { High energy flow regime. }\end{array}$ & $\begin{array}{l}\text { Distributary } \\
\text { Channel }\end{array}$ & $\begin{array}{l}21.89 \\
8-29 \\
\text { (Estimated) }\end{array}$ & $\begin{array}{l}1215 \\
510-1800 \\
\text { (Estimated) }\end{array}$ \\
\hline & $\mathrm{b}$ & $\begin{array}{l}4101- \\
4106\end{array}$ & 5 & $\begin{array}{l}\text { Fine to very fine-grained } \\
\text { sandstone, well sorted, wave } \\
\text { ripple lamination, current ripple } \\
\text { and planar current bedding. High } \\
\text { energy regime reworked by waves }\end{array}$ & Upper shoreface & $\begin{array}{l}25.25 \\
16-29 \\
\text { (Estimated) }\end{array}$ & $\begin{array}{l}1039 \\
520-1400 \\
\text { (Estimated) }\end{array}$ \\
\hline
\end{tabular}

\section{Summary}

Wells 51 and 52, sands 7, 9 and 13 are probably the same sand units since they have similar log signatures while sands units 5 and 11 both in well 52 are different since they have serrated log signatures and sand unit 11 has a funnel signature in addition. There are no Porosity and Permeability plots for sand 13, Well 51 and sands 9, 11 and 13 in Well 52 to calculate average values because of lack of lack of data. From their lithofacies and core photo description, sedimentary process and structure, textures, environment of deposition, gamma ray and resistivity logs these reservoir sands units have high quality.

Well log porosity and permeability result for all sand units in both wells have higher values than the results obtained from analyzing the core plugs, (Tables 11-15). This could be because of poor handling of the cores, technical, procedural error or probably due to the absence of bioturbation and increased or digenesis within the sand unit. The quality of a reservoir (contained reservoir fluid) is related to the textural features and depositional environment in turn control the porosity and permeability of the reservoir.

Conclusion

From the results, the reservoirs sands have been deposited in a Channel system - in the following EoD distributary channel, flood plain and fluvial channel. Only the distributary channel and fluvial depositional environments have higher reservoir quality in terms of porosity and permeability. Whereas the other reservoirs, have been deposited in a Coastal barrier systems. With the following EoD upper shoreface, lower shoreface and pro-deltaic, only the 
upper shoreface has high quality in terms of porosity and permeability. The grain size ranges of all the reservoir sands is medium to coarse $(0.5-1.75 \Phi)$, high energy of deposition and with some reservoir sands reworked by wave action. Reservoir sand units in the studied field are bioturbated except sand units 5 of Well 52 which also has a lower quality in terms of porosity and permeability. The non-reservoir units have moderate to good reservoir quality and are interpreted as flood plain, lower shoreface and prodelta shales.

Table 3 Core Description and Reservoir quality for reservoir units in Well 51

\begin{tabular}{|c|c|c|c|c|c|c|c|}
\hline Reservoir & $\begin{array}{l}\text { Reservoir } \\
\text { unit }\end{array}$ & $\begin{array}{l}\text { Depth } \\
\text { range }(\mathbf{m})\end{array}$ & $\begin{array}{l}\text { Thickness } \\
\text { (m) }\end{array}$ & $\begin{array}{l}\text { Lithologic description / sedimentary } \\
\text { processes }\end{array}$ & $\begin{array}{l}\text { Environment } \\
\text { of } \\
\text { Deposition }\end{array}$ & $\begin{array}{l}\text { Porosity } \\
(\%)\end{array}$ & $\begin{array}{l}\text { Permeability } \\
\text { (md) }\end{array}$ \\
\hline \multirow[t]{2}{*}{7} & $\mathrm{a}$ & $\begin{array}{l}3687- \\
3697\end{array}$ & 10 & $\begin{array}{l}\text { Medium to coarse grained, cross } \\
\text { bedded sandstone, poorly sorted, low } \\
\text { angle crossbedding, bioturbated- } \\
\text { ophiomorpha. High energy flow } \\
\text { regime }\end{array}$ & $\begin{array}{l}\text { Fluvial } \\
\text { channel }\end{array}$ & Good to very good & $\begin{array}{l}\text { Very good to } \\
\text { excellent }\end{array}$ \\
\hline & $\mathrm{b}$ & $\begin{array}{l}3710- \\
3718\end{array}$ & 8 & $\begin{array}{l}\text { Coarsening upward very fine to fine- } \\
\text { grained cross ripple laminated } \\
\text { sandstone, low angle crossbedding to } \\
\text { current ripple bedding. High energy } \\
\text { regime reworked by waves }\end{array}$ & Upper shoreface & Good to very good & $\begin{array}{l}\text { Very good to } \\
\text { excellent }\end{array}$ \\
\hline \multirow[t]{2}{*}{9} & $\mathrm{a}$ & $\begin{array}{l}3773- \\
3783\end{array}$ & 10 & $\begin{array}{l}\text { Pebbly coarse-medium grained cross } \\
\text { bedded fining upward sandstone, } \\
\text { planar cross bedded to low angle } \\
\text { crossbedding, poorly sorted with } \\
\text { bioturbation. Trace fossil present } \\
\text { include ophiomorpha and skolithos. } \\
\text { High energy flow regime. }\end{array}$ & $\begin{array}{l}\text { Fluvial } \\
\text { Channel }\end{array}$ & Poor to very good & $\begin{array}{l}\text { Very good to } \\
\text { excellent }\end{array}$ \\
\hline & $\mathrm{b}$ & $\begin{array}{l}3783- \\
3790\end{array}$ & 7 & $\begin{array}{l}\text { Fine to very fine-grained well sorted } \\
\text { sandstone, wave rippled to current } \\
\text { bedding and planar bedding. High } \\
\text { energy regime reworked by waves }\end{array}$ & Upper shoreface & $\begin{array}{l}\text { Negligible to very } \\
\text { good }\end{array}$ & $\begin{array}{l}\text { Moderate to } \\
\text { excellent }\end{array}$ \\
\hline \multirow[t]{2}{*}{13} & $\mathrm{a}$ & $\begin{array}{l}4088- \\
4101\end{array}$ & 12 & $\begin{array}{l}\text { Medium to coarse-grained cross } \\
\text { bedded blocky sandstone, low angle } \\
\text { crossbedding moderate to poorly } \\
\text { sorted, bioturbated, present is } \\
\text { ophiomorpha and planolites. High } \\
\text { energy flow regime. }\end{array}$ & $\begin{array}{l}\text { Distributary } \\
\text { Channel }\end{array}$ & $\begin{array}{l}\text { Poor to very good } \\
\text { (Estimated) }\end{array}$ & $\begin{array}{l}\text { Very good to } \\
\text { excellent } \\
\text { (Estimated) }\end{array}$ \\
\hline & $\mathrm{b}$ & $\begin{array}{l}4101- \\
4106\end{array}$ & 5 & $\begin{array}{l}\text { Fine to very fine-grained sandstone, } \\
\text { well sorted, wave ripple lamination, } \\
\text { current ripple and planar current } \\
\text { bedding. High energy regime } \\
\text { reworked by waves }\end{array}$ & Upper shoreface & $\begin{array}{l}\text { Negligible to very } \\
\text { good } \\
\text { (Estimated) }\end{array}$ & $\begin{array}{l}\text { Moderate to } \\
\text { excellent } \\
\text { (Estimated) }\end{array}$ \\
\hline
\end{tabular}


Table 4 Core Description, Depositional Environment, Porosity and Permeability of Non-Reservoir units in Well 51

\begin{tabular}{|c|c|c|c|c|c|c|c|}
\hline Reservoir & $\begin{array}{l}\text { Reservoir } \\
\text { unit }\end{array}$ & $\begin{array}{l}\text { Depth } \\
\text { range (m) }\end{array}$ & $\begin{array}{l}\text { Thickness } \\
\text { (m) }\end{array}$ & $\begin{array}{l}\text { Lithologic description / } \\
\text { sedimentary processes }\end{array}$ & $\begin{array}{l}\text { Environment } \\
\text { of } \\
\text { Deposition }\end{array}$ & $\begin{array}{l}\text { Porosity } \\
(\%)\end{array}$ & $\begin{array}{l}\text { Permeability } \\
\text { (md) }\end{array}$ \\
\hline \multirow[t]{2}{*}{7} & $\mathrm{a}$ & $\begin{array}{l}3697- \\
3704\end{array}$ & 7 & $\begin{array}{l}\text { Heteroliths, planar laminated } \\
\text { slightly mudstone trace fossils } \\
\text { present include ophiomorpha and } \\
\text { planolites. Low energy regime } \\
\text { with periodic influx of silt. }\end{array}$ & $\begin{array}{l}\text { Lower } \\
\text { shoreface }\end{array}$ & $\begin{array}{l}17 \\
8-28\end{array}$ & $\begin{array}{l}288.21 \\
0.1-1000\end{array}$ \\
\hline & $\mathrm{b}$ & $\begin{array}{l}3704- \\
3710\end{array}$ & 6 & $\begin{array}{l}\text { Dark grey silty shales. Low } \\
\text { energy. }\end{array}$ & Prodelta & $\begin{array}{l}13.67 \\
12-16\end{array}$ & $\begin{array}{l}83.42 \\
0.1-500\end{array}$ \\
\hline \multirow[t]{2}{*}{9} & $\mathrm{a}$ & $\begin{array}{l}3764- \\
3773\end{array}$ & 9 & $\begin{array}{l}\text { Wavy bedded heterolithic and } \\
\text { bioturbated, trace fossils present } \\
\text { include ophiomorpha, planolites } \\
\text { and skolithos. Low energy regime } \\
\text { with periodic influx of silt. }\end{array}$ & $\begin{array}{l}\text { Lower } \\
\text { shoreface }\end{array}$ & $\begin{array}{l}16.7 \\
9-25\end{array}$ & $\begin{array}{l}241.1 \\
0.1-1000\end{array}$ \\
\hline & $\mathrm{b}$ & $\begin{array}{l}3791- \\
3794\end{array}$ & 3 & $\begin{array}{l}\text { Very fine-grained ripple } \\
\text { laminated, interlaminated clay, } \\
\text { intensely bioturbated. Trace fossil } \\
\text { present include skolithos and } \\
\text { planolites. Low energy regime } \\
\text { with periodic influx of silt. }\end{array}$ & $\begin{array}{l}\text { Lower } \\
\text { shoreface }\end{array}$ & $\begin{array}{l}18.5 \\
12-25\end{array}$ & $\begin{array}{l}278.5 \\
7-600\end{array}$ \\
\hline \multirow[t]{2}{*}{13} & $\mathrm{a}$ & $\begin{array}{l}4078- \\
4088\end{array}$ & 10 & $\begin{array}{l}\text { Siltstone, light brownish grey, } \\
\text { wave ripple laminated, inter } \\
\text { laminated with mudstone. } \\
\text { Heterolithic wavy bedding. Trace } \\
\text { fossil present is planolites. Low } \\
\text { energy regime with periodic } \\
\text { influx of silt. }\end{array}$ & $\begin{array}{l}\text { Lower } \\
\text { shoreface }\end{array}$ & $\begin{array}{l}16.7 \\
9-25 \\
\text { (Estimated) }\end{array}$ & $\begin{array}{l}241.1 \\
0.1-1000 \\
\text { (Estimated) }\end{array}$ \\
\hline & $\mathrm{b}$ & $\begin{array}{l}4121- \\
4129\end{array}$ & 8 & $\begin{array}{l}\text { Dark grey silty shales. Low } \\
\text { energy. }\end{array}$ & Prodelta & $\begin{array}{l}13.67 \\
12-16 \\
\text { (Estimated) }\end{array}$ & $\begin{array}{l}83.42 \\
0.1 \quad-500 \\
(\text { Estimated) }\end{array}$ \\
\hline
\end{tabular}


Table 5 Core Description, Depositional Environment and Reservoir Quality of the Non-Reservoir units in Well 51

\begin{tabular}{|c|c|c|c|c|c|c|c|}
\hline Reservoir & $\begin{array}{l}\text { Reservoir } \\
\text { unit }\end{array}$ & $\begin{array}{l}\text { Depth } \\
\text { range (m) }\end{array}$ & $\begin{array}{l}\text { Thickness } \\
\text { (m) }\end{array}$ & $\begin{array}{l}\text { Lithologic description / } \\
\text { sedimentary processes }\end{array}$ & $\begin{array}{l}\text { Environment } \\
\text { of } \\
\text { Deposition }\end{array}$ & $\begin{array}{l}\text { Porosity } \\
(\%)\end{array}$ & $\begin{array}{l}\text { Permeability } \\
\text { (md) }\end{array}$ \\
\hline \multirow[t]{2}{*}{7} & $\mathrm{a}$ & $\begin{array}{l}3697- \\
3704\end{array}$ & 7 & $\begin{array}{l}\text { Heteroliths, planar laminated } \\
\text { slightly mudstone trace fossils } \\
\text { present include ophiomorpha and } \\
\text { planolites. Low energy regime } \\
\text { with periodic influx of silt. } \\
\end{array}$ & $\begin{array}{l}\text { Lower } \\
\text { shoreface }\end{array}$ & Poor to very good & $\begin{array}{l}\text { Very good to } \\
\text { Excellent }\end{array}$ \\
\hline & $\mathrm{b}$ & $\begin{array}{l}3704- \\
3710\end{array}$ & 6 & $\begin{array}{l}\text { Dark grey silty shales. Low } \\
\text { energy. }\end{array}$ & Prodelta & Good & $\begin{array}{l}\text { Good to very } \\
\text { good }\end{array}$ \\
\hline \multirow[t]{2}{*}{9} & $\mathrm{a}$ & $\begin{array}{l}3764- \\
3773\end{array}$ & 9 & $\begin{array}{l}\text { Wavy bedded heterolithic and } \\
\text { bioturbated, trace fossils present } \\
\text { include ophiomorpha, planolites } \\
\text { and skolithos. Low energy regime } \\
\text { with periodic influx of silt. }\end{array}$ & $\begin{array}{l}\text { Lower } \\
\text { shoreface }\end{array}$ & Poor to very good & $\begin{array}{l}\text { Good to very } \\
\text { good }\end{array}$ \\
\hline & $\mathrm{b}$ & $\begin{array}{l}3791- \\
3794\end{array}$ & 3 & $\begin{array}{l}\text { Very fine-grained ripple } \\
\text { laminated, interlaminated clay, } \\
\text { intensely bioturbated. Trace fossil } \\
\text { present include skolithos and } \\
\text { planolites. Low energy regime } \\
\text { with periodic influx of silt. }\end{array}$ & $\begin{array}{l}\text { Lower } \\
\text { shoreface }\end{array}$ & Good to very good & Poor to very good \\
\hline \multirow[t]{2}{*}{13} & $\mathrm{a}$ & $\begin{array}{l}4078- \\
4088\end{array}$ & 10 & $\begin{array}{l}\text { Siltstone, light brownish grey, } \\
\text { wave ripple laminated, inter } \\
\text { laminated with mudstone. } \\
\text { Heterolithic wavy bedding. Trace } \\
\text { fossil present is planolites. Low } \\
\text { energy regime with periodic } \\
\text { influx of silt. }\end{array}$ & $\begin{array}{l}\text { Lower } \\
\text { shoreface }\end{array}$ & Poor to very good & Poor to very good \\
\hline & $\mathrm{b}$ & $\begin{array}{l}4121- \\
4129\end{array}$ & 8 & $\begin{array}{l}\text { Dark grey silty shales. Low } \\
\text { energy. }\end{array}$ & Prodelta & Poor to good & Poor to very good \\
\hline
\end{tabular}


Table 6 Core Description, Deposition Environment, Porosity and Permeability for reservoir units in Well 52

\begin{tabular}{|c|c|c|c|c|c|c|c|}
\hline Reservoir & $\begin{array}{l}\text { Reservoir } \\
\text { unit }\end{array}$ & $\begin{array}{l}\text { Depth } \\
\text { range (m) }\end{array}$ & $\begin{array}{l}\text { Thicknes } \\
\text { s (m) }\end{array}$ & $\begin{array}{l}\text { Lithologic description / } \\
\text { sedimentary processes }\end{array}$ & $\begin{array}{l}\text { Environment } \\
\text { of } \\
\text { Deposition }\end{array}$ & $\begin{array}{l}\text { Porosity } \\
\text { Mean and } \\
\text { Range }(\%)\end{array}$ & $\begin{array}{l}\text { Permeability } \\
\text { Mean and range } \\
\text { (md) }\end{array}$ \\
\hline \multirow[t]{2}{*}{5} & $\mathrm{a}$ & $\begin{array}{l}3324 \\
3328\end{array}$ & 4 & $\begin{array}{l}\text { Medium to coarse grained, cross } \\
\text { bedded sandstone, moderate to } \\
\text { poorly sorted and laminated. High } \\
\text { energy flow regime }\end{array}$ & $\begin{array}{l}\text { Fluvial } \\
\text { channel }\end{array}$ & $\begin{array}{l}18.44 \\
8-32\end{array}$ & $\begin{array}{l}747.67 \\
8-4000\end{array}$ \\
\hline & $\mathrm{b}$ & $\begin{array}{l}3328- \\
3335\end{array}$ & 7 & $\begin{array}{l}\text { Coarsening upward fine to very } \\
\text { fine-grained well sorted } \\
\text { sandstone. High energy regime } \\
\text { reworked by waves }\end{array}$ & Upper shoreface & $\begin{array}{l}22.71 \\
6-30\end{array}$ & $\begin{array}{l}6198.53 \\
12-80000\end{array}$ \\
\hline \multirow[t]{2}{*}{7} & $\mathrm{a}$ & $\begin{array}{l}3324- \\
3328\end{array}$ & 4 & $\begin{array}{l}\text { Medium to coarse grained, } \\
\text { moderate to poorly sorted, and } \\
\text { laminated. High energy flow } \\
\text { regime }\end{array}$ & $\begin{array}{l}\text { Fluvial } \\
\text { Channel }\end{array}$ & $\begin{array}{l}18.82 \\
8-32\end{array}$ & $\begin{array}{l}902.2 \\
0.6-600\end{array}$ \\
\hline & $\mathrm{b}$ & $\begin{array}{l}3328- \\
3334\end{array}$ & 6 & $\begin{array}{l}\text { Coarsening upward fine to very } \\
\text { fine-grained cross rippled, } \\
\text { laminated, trough bedded, well } \\
\text { sorted sandstone. High energy } \\
\text { regime reworked by waves }\end{array}$ & Upper shoreface & $\begin{array}{l}22.76 \\
10-30\end{array}$ & $\begin{array}{l}915.32 \\
11-10500\end{array}$ \\
\hline \multirow[t]{2}{*}{9} & $\mathrm{a}$ & $\begin{array}{l}3444- \\
3468\end{array}$ & 24 & $\begin{array}{l}\text { Medium to coarse-grained cross } \\
\text { bedded blocky sandstone, low } \\
\text { angle crossbedding moderate to } \\
\text { poorly sorted, bioturbated, present } \\
\text { is ophiomorpha and planolites. } \\
\text { High energy flow regime. }\end{array}$ & $\begin{array}{l}\text { Distributary } \\
\text { Channel }\end{array}$ & $\begin{array}{l}21.89 \\
4-28\end{array}$ & $\begin{array}{l}9025.04 \\
0.7-19000\end{array}$ \\
\hline & $\mathrm{b}$ & $\begin{array}{l}3472- \\
3480\end{array}$ & 8 & $\begin{array}{l}\text { Medium to coarse-grained cross } \\
\text { bedded blocky sandstone, low } \\
\text { angle crossbedding moderate to } \\
\text { poorly sorted, bioturbated, present } \\
\text { is ophiomorpha and planolites. } \\
\text { High energy flow regime. }\end{array}$ & $\begin{array}{l}\text { Distributary } \\
\text { Channel }\end{array}$ & $\begin{array}{l}25.25 \\
16-29 \\
\text { (Estimated) }\end{array}$ & $\begin{array}{l}1039 \\
520-1400 \\
\text { (Estimated) }\end{array}$ \\
\hline \multirow[t]{2}{*}{13} & $\mathrm{a}$ & $\begin{array}{l}3790- \\
3793\end{array}$ & 3 & $\begin{array}{l}\text { Coarsening upward fine to very } \\
\text { fine-grained cross rippled, } \\
\text { laminated, trough bedded, well } \\
\text { sorted sandstone. High energy } \\
\text { regime reworked by waves }\end{array}$ & Upper shoreface & $\begin{array}{l}22.76 \\
10-30 \\
\text { (Estimated) }\end{array}$ & $\begin{array}{l}915.32 \\
11-10500 \\
\text { (Estimated) }\end{array}$ \\
\hline & $\mathrm{b}$ & & & $\begin{array}{l}\text { Pebbly-coarse medium grained } \\
\text { cross bedded fining upward } \\
\text { sandstone, planar cross bedded to } \\
\text { low angle crossbedding, poorly } \\
\text { sorted with bioturbation. Trace } \\
\text { fossil present include } \\
\text { ophiomorpha and skolithos. High } \\
\text { energy flow regime. }\end{array}$ & $\begin{array}{l}\text { Fluvial } \\
\text { channel }\end{array}$ & $\begin{array}{l}18.44 \\
8-32 \\
\text { (Estimated) }\end{array}$ & $\begin{array}{l}747.67 \\
8-4000 \\
\text { (Estimated) }\end{array}$ \\
\hline
\end{tabular}


Table 7 Core Description and Reservoir quality for reservoir units in Well 52

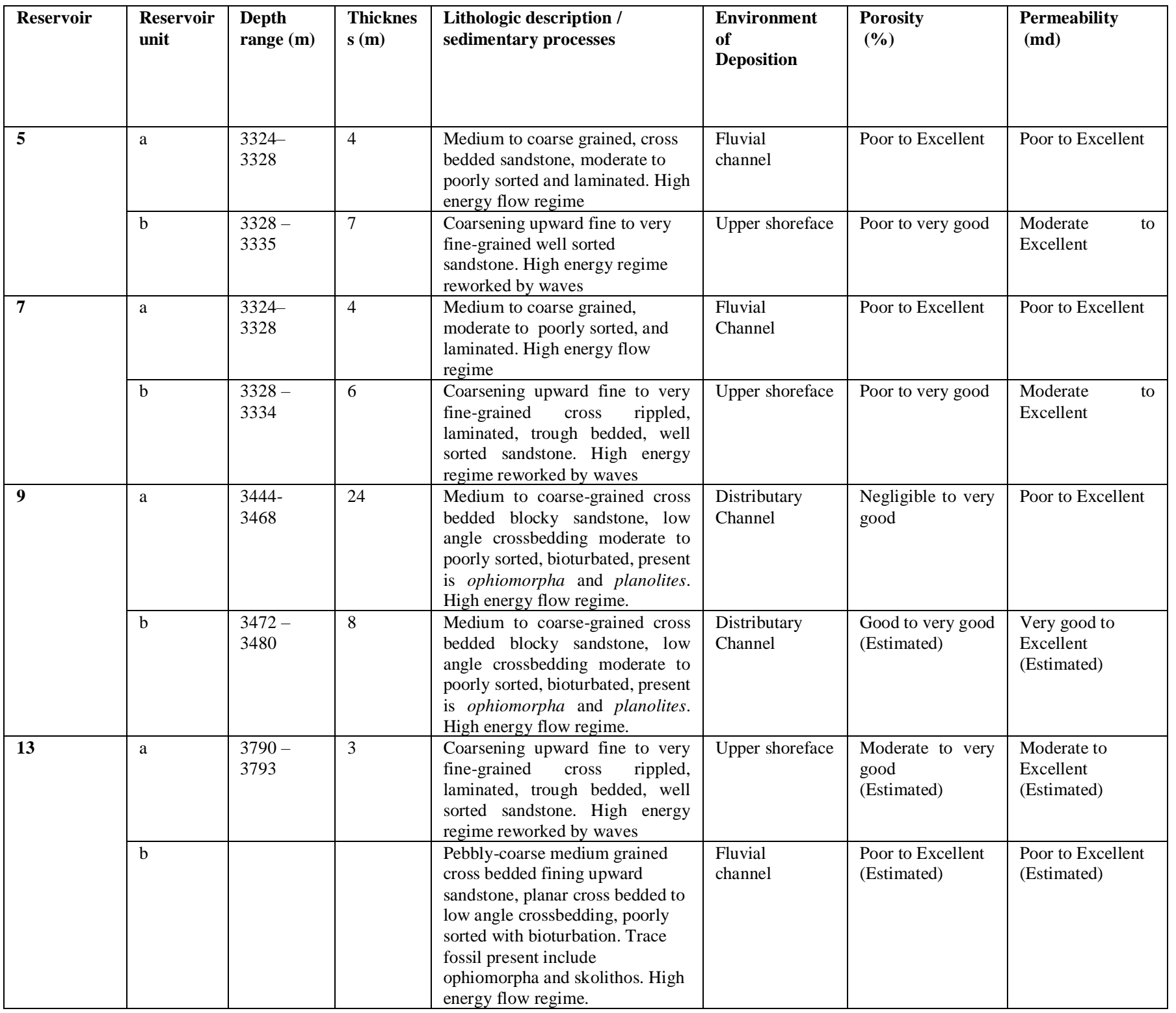


Table 8 Core Description, Deposition Environment, Porosity and Permeability for Non-reservoir units in Well 52

\begin{tabular}{|c|c|c|c|c|c|c|c|}
\hline Reservoir & $\begin{array}{l}\text { Reservoir } \\
\text { unit }\end{array}$ & $\begin{array}{l}\text { Depth } \\
\text { range (m) }\end{array}$ & $\begin{array}{l}\text { Thicknes } \\
\text { s (m) }\end{array}$ & $\begin{array}{l}\text { Lithologic description / } \\
\text { sedimentary processes }\end{array}$ & $\begin{array}{l}\text { Environment } \\
\text { of } \\
\text { Deposition }\end{array}$ & $\begin{array}{l}\text { Porosity } \\
\text { Mean and } \\
\text { Range (\%) }\end{array}$ & $\begin{array}{l}\text { Permeability } \\
\text { Mean and range } \\
\text { (md) }\end{array}$ \\
\hline \multirow[t]{3}{*}{5} & $\mathrm{a}$ & $\begin{array}{l}3322- \\
3324\end{array}$ & 2 & $\begin{array}{l}\text { Sandy mudstone, grey and } \\
\text { intensely bioturbated, trace fossil } \\
\text { present is skolithos. Low energy }\end{array}$ & Flood plain & $\begin{array}{l}12.11 \\
3-23\end{array}$ & $\begin{array}{l}33.00 \\
10-120\end{array}$ \\
\hline & $\mathrm{b}$ & $\begin{array}{l}3335- \\
3339\end{array}$ & 4 & $\begin{array}{l}\text { Heteroliths, alternation of sandy } \\
\text { and muddy heteroliths, planar } \\
\text { laminated mudstones, moderately } \\
\text { bioturbated. Trace fossils present } \\
\text { include ophiomorpha and } \\
\text { skolithos. Low energy regime } \\
\text { with periodic influx of silt }\end{array}$ & $\begin{array}{l}\text { Lower } \\
\text { shoreface }\end{array}$ & $\begin{array}{l}6 \\
8-28\end{array}$ & $\begin{array}{l}288.21 \\
0.1-1000\end{array}$ \\
\hline & $\mathrm{c}$ & $\begin{array}{l}3339- \\
3341\end{array}$ & 2 & $\begin{array}{l}\text { Dark grey silty shales. Low } \\
\text { energy. }\end{array}$ & Prodelta & $\begin{array}{l}12.11 \\
3-23\end{array}$ & $\begin{array}{l}33.00 \\
10-120\end{array}$ \\
\hline 7 & $\mathrm{a}$ & $\begin{array}{l}3334- \\
3342\end{array}$ & 8 & $\begin{array}{l}\text { Heteroliths, alternation of sandy } \\
\text { and muddy heteroliths, planar } \\
\text { laminated mudstones, moderately } \\
\text { bioturbated. Trace fossils present } \\
\text { include ophiomorpha and } \\
\text { skolithos. Low energy regime } \\
\text { with periodic influx of silt }\end{array}$ & Flood plain & $\begin{array}{l}12.11 \\
3-23\end{array}$ & $\begin{array}{l}33.00 \\
10-120\end{array}$ \\
\hline \multirow[t]{4}{*}{9} & $a$ & $\begin{array}{l}3468- \\
3472\end{array}$ & 8 & $\begin{array}{l}\text { Dark grey silty shales. Low } \\
\text { energy. }\end{array}$ & Prodelta & $\begin{array}{l}21.89 \\
4-28\end{array}$ & $\begin{array}{l}9025.04 \\
0.7-19000\end{array}$ \\
\hline & $\mathrm{b}$ & $\begin{array}{l}3555- \\
3559\end{array}$ & 4 & $\begin{array}{l}\text { Wavy bedded heterolithic and } \\
\text { bioturbated, trace fossils present } \\
\text { include ophiomorpha, planolites } \\
\text { and skolithos. Low energy regime } \\
\text { with periodic influx of silt. }\end{array}$ & $\begin{array}{l}\text { Lower } \\
\text { shoreface }\end{array}$ & $\begin{array}{l}12.11 \\
3-23\end{array}$ & $\begin{array}{l}33.00 \\
10-120\end{array}$ \\
\hline & $\mathrm{c}$ & $\begin{array}{l}3698- \\
3708\end{array}$ & 10 & $\begin{array}{l}\text { Heteroliths, alternation of sandy } \\
\text { and muddy heteroliths, planar } \\
\text { laminated mudstones, moderately } \\
\text { bioturbated. Trace fossils present } \\
\text { include ophiomorpha and } \\
\text { skolithos. Low energy regime } \\
\text { with periodic influx of silt }\end{array}$ & $\begin{array}{l}\text { Lower } \\
\text { shoreface }\end{array}$ & $\begin{array}{l}12.11 \\
3-23\end{array}$ & $\begin{array}{l}33.00 \\
10-120\end{array}$ \\
\hline & $\mathrm{d}$ & $\begin{array}{l}3708- \\
3710\end{array}$ & 2 & $\begin{array}{l}\text { Dark grey silty shales. Low } \\
\text { energy. }\end{array}$ & Prodelta & $\begin{array}{l}12.11 \\
3-23\end{array}$ & $\begin{array}{l}33.00 \\
10-120\end{array}$ \\
\hline \multirow[t]{2}{*}{13} & $\mathrm{a}$ & $\begin{array}{l}3793- \\
3796\end{array}$ & 3 & $\begin{array}{l}\text { Heteroliths, planar laminated } \\
\text { slightly mudstone trace fossils } \\
\text { present include ophiomorpha and } \\
\text { planolites. Low energy regime } \\
\text { with periodic influx of silt. }\end{array}$ & $\begin{array}{l}\text { Lower } \\
\text { shoreface }\end{array}$ & $\begin{array}{l}12.11 \\
3-23\end{array}$ & $\begin{array}{l}33.00 \\
10-120\end{array}$ \\
\hline & $\mathrm{b}$ & $\begin{array}{l}3798- \\
3810\end{array}$ & 2 & $\begin{array}{l}\text { Dark grey silty shales. Low } \\
\text { energy. }\end{array}$ & Prodelta & $\begin{array}{r}12.11 \\
3-23 \\
\end{array}$ & $\begin{array}{l}33.00 \\
10-120 \\
\end{array}$ \\
\hline
\end{tabular}


Table 9 Core Description, Deposition Environment and Reservoir Quality of the Non-reservoir units in Well 52

\begin{tabular}{|c|c|c|c|c|c|c|c|}
\hline Reservoir & $\begin{array}{l}\text { Reservoir } \\
\text { unit }\end{array}$ & $\begin{array}{l}\text { Depth } \\
\text { range (m) }\end{array}$ & $\begin{array}{l}\text { Thicknes } \\
\text { s (m) }\end{array}$ & $\begin{array}{l}\text { Lithologic description / } \\
\text { sedimentary processes }\end{array}$ & $\begin{array}{l}\text { Environment } \\
\text { of } \\
\text { Deposition }\end{array}$ & $\begin{array}{l}\text { Porosity } \\
\text { Mean and } \\
\text { Range (\%) }\end{array}$ & $\begin{array}{l}\text { Permeability } \\
\text { Mean and range } \\
\text { (md) }\end{array}$ \\
\hline \multirow[t]{3}{*}{5} & $\mathrm{a}$ & $\begin{array}{l}3322- \\
3324\end{array}$ & 2 & $\begin{array}{l}\text { Sandy mudstone, grey and } \\
\text { intensely bioturbated, trace fossil } \\
\text { present is skolithos. Low energy }\end{array}$ & Flood plain & $\begin{array}{l}12.11 \\
3-23\end{array}$ & $\begin{array}{l}33.00 \\
10-120\end{array}$ \\
\hline & $\mathrm{b}$ & $\begin{array}{l}3335- \\
3339\end{array}$ & 4 & $\begin{array}{l}\text { Heteroliths, alternation of sandy } \\
\text { and muddy heteroliths, planar } \\
\text { laminated mudstones, moderately } \\
\text { bioturbated. Trace fossils present } \\
\text { include ophiomorpha and } \\
\text { skolithos. Low energy regime } \\
\text { with periodic influx of silt }\end{array}$ & $\begin{array}{l}\text { Lower } \\
\text { shoreface }\end{array}$ & $\begin{array}{l}6 \\
8-28\end{array}$ & $\begin{array}{l}288.21 \\
0.1-1000\end{array}$ \\
\hline & $\mathrm{c}$ & $\begin{array}{l}3339- \\
3341\end{array}$ & 2 & $\begin{array}{l}\text { Dark grey silty shales. Low } \\
\text { energy. }\end{array}$ & Prodelta & $\begin{array}{l}12.11 \\
3-23\end{array}$ & $\begin{array}{l}33.00 \\
10-120\end{array}$ \\
\hline 7 & $\mathrm{a}$ & $\begin{array}{l}3334- \\
3342\end{array}$ & 8 & $\begin{array}{l}\text { Heteroliths, alternation of sandy } \\
\text { and muddy heteroliths, planar } \\
\text { laminated mudstones, moderately } \\
\text { bioturbated. Trace fossils present } \\
\text { include ophiomorpha and } \\
\text { skolithos. Low energy regime } \\
\text { with periodic influx of silt }\end{array}$ & $\begin{array}{l}\text { Lower } \\
\text { shoreface }\end{array}$ & $\begin{array}{l}12.11 \\
3-23\end{array}$ & $\begin{array}{l}33.00 \\
10-120\end{array}$ \\
\hline \multirow[t]{4}{*}{9} & $\mathrm{a}$ & $\begin{array}{l}3468- \\
3472\end{array}$ & 8 & $\begin{array}{l}\text { Dark grey silty shales. Low } \\
\text { energy. }\end{array}$ & Prodelta & $\begin{array}{l}21.89 \\
4-28\end{array}$ & $\begin{array}{l}9025.04 \\
0.7-19000\end{array}$ \\
\hline & $\mathrm{b}$ & $\begin{array}{l}3555- \\
3559\end{array}$ & 4 & $\begin{array}{l}\text { Wavy bedded heterolithic and } \\
\text { bioturbated, trace fossils present } \\
\text { include ophiomorpha, planolites } \\
\text { and skolithos. Low energy regime } \\
\text { with periodic influx of silt. }\end{array}$ & $\begin{array}{l}\text { Lower } \\
\text { shoreface }\end{array}$ & $\begin{array}{l}12.11 \\
3-23\end{array}$ & $\begin{array}{l}33.00 \\
10-120\end{array}$ \\
\hline & $\mathrm{c}$ & $\begin{array}{l}3698- \\
3708\end{array}$ & 10 & $\begin{array}{l}\text { Heteroliths, alternation of sandy } \\
\text { and muddy heteroliths, planar } \\
\text { laminated mudstones, moderately } \\
\text { bioturbated. Trace fossils present } \\
\text { include ophiomorpha and } \\
\text { skolithos. Low energy regime } \\
\text { with periodic influx of silt }\end{array}$ & $\begin{array}{l}\text { Lower } \\
\text { shoreface }\end{array}$ & $\begin{array}{l}12.11 \\
3-23\end{array}$ & $\begin{array}{l}33.00 \\
10-120\end{array}$ \\
\hline & $\mathrm{d}$ & $\begin{array}{l}3708- \\
3710\end{array}$ & 2 & $\begin{array}{l}\text { Dark grey silty shales. Low } \\
\text { energy. }\end{array}$ & Prodelta & $\begin{array}{l}12.11 \\
3-23\end{array}$ & $\begin{array}{l}33.00 \\
10-120\end{array}$ \\
\hline \multirow[t]{2}{*}{13} & $\mathrm{a}$ & $\begin{array}{l}3793- \\
3796\end{array}$ & 3 & $\begin{array}{l}\text { Heteroliths, planar laminated } \\
\text { slightly mudstone trace fossils } \\
\text { present include ophiomorpha and } \\
\text { planolites. Low energy regime } \\
\text { with periodic influx of silt. }\end{array}$ & $\begin{array}{l}\text { Lower } \\
\text { shoreface }\end{array}$ & $\begin{array}{l}12.11 \\
3-23\end{array}$ & $\begin{array}{l}33.00 \\
10-120\end{array}$ \\
\hline & $\mathrm{b}$ & $\begin{array}{l}3798- \\
3810\end{array}$ & 2 & $\begin{array}{l}\text { Dark grey silty shales. Low } \\
\text { energy. }\end{array}$ & Prodelta & $\begin{array}{l}12.11 \\
3-23\end{array}$ & $\begin{array}{l}33.00 \\
10-120\end{array}$ \\
\hline
\end{tabular}


Table 10 Sedimentology and depositional environment of Sand Units in Well 51 Gabro Field.

\begin{tabular}{|l|l|l|l|l|l|l|}
\hline \multicolumn{7}{|c|}{ WELL 51 } \\
\hline Reservoir & $\begin{array}{l}\text { Thickness } \\
(\mathrm{m})\end{array}$ & Sorting & $\begin{array}{l}\text { Grain size } \\
(\Phi)\end{array}$ & Log shape & $\begin{array}{l}\text { Depositional } \\
\text { Environment }\end{array}$ & $\begin{array}{l}\text { Porosity- } \\
\text { permeability }\end{array}$ \\
\hline 7 & 13 & $\begin{array}{l}\text { Poorly } \\
\text { sorted }\end{array}$ & $1.75--.25$ & Cylindrical & $\begin{array}{l}\text { Fluvial } \\
\text { channel }\end{array}$ & Good \\
\hline 9 & 18.5 & $\begin{array}{l}\text { Well } \\
\text { sorted }\end{array}$ & $-2-4$ & $\begin{array}{l}\text { Funnel } \\
\text { shaped }\end{array}$ & $\begin{array}{l}\text { Upper } \\
\text { shoreface }\end{array}$ & Good \\
\hline 13 & 13 & $\begin{array}{l}\text { Poorly } \\
\text { sorted }\end{array}$ & $-.25-1.75$ & Blocky & $\begin{array}{l}\text { Distributary } \\
\text { channel }\end{array}$ & Excellent \\
\cline { 2 - 7 } & 9.4 & $\begin{array}{l}\text { Well } \\
\text { sorted }\end{array}$ & $2-4$ & $\begin{array}{l}\text { Funnel } \\
\text { shaped }\end{array}$ & $\begin{array}{l}\text { Upper } \\
\text { shoreface }\end{array}$ & Excellent \\
\hline
\end{tabular}

Table 12 Sedimentology and depositional environment in Well 52, Gabro Field.

\begin{tabular}{|l|l|l|l|l|l|l|}
\hline \multicolumn{7}{|c|}{ WELL 52 } \\
\hline Reservoir & $\begin{array}{l}\text { Thickness } \\
(\mathrm{m})\end{array}$ & Sorting & $\begin{array}{l}\text { Grain } \\
\text { size }(\Phi)\end{array}$ & Log shape & $\begin{array}{l}\text { Depositional } \\
\text { Environment }\end{array}$ & $\begin{array}{l}\text { Porosity- } \\
\text { permeability }\end{array}$ \\
\hline 5 & 12 & Well sorted & $\begin{array}{l}1.75 \text { to }- \\
.25\end{array}$ & $\begin{array}{l}\text { Funnel } \\
\text { shaped }\end{array}$ & $\begin{array}{l}\text { Upper } \\
\text { shoreface }\end{array}$ & Good \\
\hline 7 & 23 & $\begin{array}{l}\text { Moderately } \\
\text { sorted }\end{array}$ & 1.75 to - & Blocky & $\begin{array}{l}\text { Distributary } \\
\text { channel }\end{array}$ & Excellent \\
\hline 9 & 14 & $\begin{array}{l}\text { Moderately } \\
\text { sorted }\end{array}$ & 1.75 to - & Blocky & $\begin{array}{l}\text { Distributary } \\
\text { channel }\end{array}$ & Excellent \\
\hline 11 & 7.5 & Well sorted & 1.75 to 4 & $\begin{array}{l}\text { Funnel } \\
\text { shaped }\end{array}$ & $\begin{array}{l}\text { Upper } \\
\text { shoreface }\end{array}$ & Good \\
\hline 13 & 13 & $\begin{array}{l}\text { Poorly } \\
\text { sorted }\end{array}$ & -2 to -1 & Blocky & $\begin{array}{l}\text { Fluvial } \\
\text { channel }\end{array}$ & Very Good \\
\hline
\end{tabular}

Table 13 Reservoir quality of Well 51, Gabro Field (Core plug values) - (Rider, 1986; Etu - Efeotor, (2007)

\begin{tabular}{|l|l|l|l|l|}
\hline \multirow{2}{*}{ Sand } & \multicolumn{2}{|c|}{ Permeability (md) } & \multicolumn{2}{c|}{ Porosity (\%) } \\
\cline { 2 - 5 } & Quantitative & Qualitative & Quantitative & Qualitative \\
\hline 7 & 980.0 & Good & 23.5 & Very good \\
\hline 9 & 950.0 & Good & 16.0 & Good \\
\hline 13 & & - & - & Good - Very good \\
\hline Range & $950.0-980.0$ & & $16.0-23.5$ & Good \\
\hline average & 965.0 & & 19.75 & \\
\hline
\end{tabular}


Table 14 Reservoir quality of Well 51, Gabro Field (based on Well log) - (Rider, 1986; Etu - Efeotor, (2007)

\begin{tabular}{|l|l|l|l|l|}
\hline \multirow{2}{*}{ Sand } & \multicolumn{2}{|c|}{ Permeability $(\mathrm{mD})$} & \multicolumn{2}{c|}{ Porosity $(\%)$} \\
\cline { 2 - 5 } & Quantitative & Qualitative & Quantitative & Qualitative \\
\hline 7 & $1,978.7$ & Excellent & 24.8 & Very Good \\
\hline 9 & 1238.3 & Excellent & 21.8 & Very Good \\
\hline 13 & 1565.2 & Excellent & 22.4 & Very Good \\
\hline Range & $1238.3-1978.7$ & Excellent & $21.8-24.8$ & Very good \\
\hline Average & 1594.1 & Excellent & 22.73 & Very good \\
\hline
\end{tabular}

Table 15 Reservoir quality of Well 52 Gabro Field (based on Well log) - (Rider, 1986; Etu - Efeotor, (2007)

\begin{tabular}{|l|l|l|l|l|}
\hline \multirow{2}{*}{ Sand } & \multicolumn{2}{|c|}{ Permeability $(\mathrm{mD})$} & \multicolumn{2}{c|}{ Porosity (\%) } \\
\cline { 2 - 5 } & Quantitative & Qualitative & Quantitative & Qualitative \\
\hline 5 & 1009.7 & Very Good & 14.8 & Good \\
\hline 7 & 1156.3 & Excellent & 17.9 & Good \\
\hline 9 & 1068.0 & Excellent & 18.5 & Good \\
\hline 11 & 1317.4 & Excellent & 16.7 & Good \\
\hline 13 & 2330.0 & Excellent & 28.2 & Very Good \\
\hline Range & $1009.7-2330.0$ & Good - excellent & $14.8-28.2$ & Good - very good \\
\hline Average & 1376.24 & Excellent & 19.22 & good \\
\hline
\end{tabular}
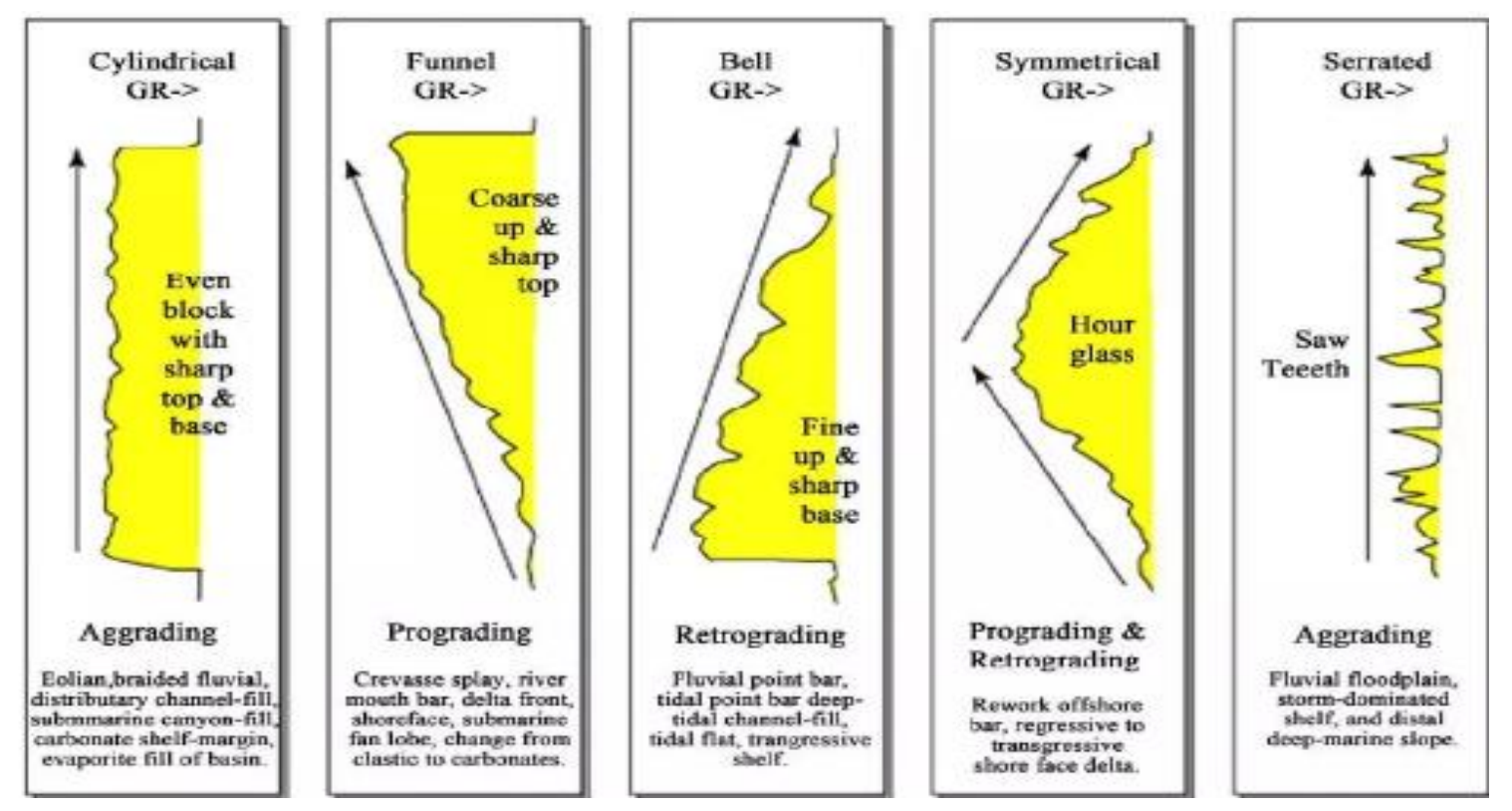

Figure 6 GR Log response for different environments - shows how vertical grain size profile of sandstone used to interpret facies (Rider, 1986;) 


\begin{tabular}{|c|c|c|c|c|}
\hline & $\begin{array}{l}\text { Well log } \\
\text { signatures }\end{array}$ & $\begin{array}{l}\text { Log patterns/ } \\
\text { motifs } \\
0 \quad 150\end{array}$ & Lithofacies Descriptions & Lithofacies Interpretations \\
\hline A & $\begin{array}{l}\text { Low serrated } \\
\text { gamma }\end{array}$ & I $\quad 20 \mathrm{~m}$ & $\begin{array}{l}\text { Stacked up of bell shape (coastal } \\
\text { barrier bar) and funnel shape (fluvi- } \\
\text { al/tidally influence channel sand }\end{array}$ & $\begin{array}{l}\text { Undifferentiated Continental } \\
\text { deposits (Benin Formation) }\end{array}$ \\
\hline $\mathrm{Cl}_{1}$ & $\begin{array}{l}\text { Blocky and } \\
\text { dirtying } \\
\text { upwards }\end{array}$ & $I_{20 \mathrm{~m}}^{\frac{2}{2 \frac{2}{2}}}$ & $\begin{array}{l}\text { Bow/ bell shape morphology, shows } \\
\text { some high gamma values } \\
\text { anormalies, the top units marked } \\
\text { the transgressive surfaces while the } \\
\text { basal marked the sequence boundaries. }\end{array}$ & $\begin{array}{l}\text { Tidal/Fluvial channel } \\
\text { fills }\end{array}$ \\
\hline D & $\begin{array}{l}\text { Gradual } \\
\text { cleaning } \\
\text { upwards }\end{array}$ & $\begin{array}{l}\tau \\
I 20 \mathrm{~m}\end{array}$ & $\begin{array}{l}\text { Funnel shape morphology and less } \\
\text { blocky than coastal barrier bar. The } \\
\text { upper contact is defined by the } \\
\text { transgressive/flooding surfaces } \\
\text { while the basal part is defined by } \\
\text { shallow marine deepening. }\end{array}$ & Upper shoreface sand \\
\hline$E \& F$ & $\begin{array}{l}\text { High serrated } \\
\text { gamma }\end{array}$ & I $20 \mathrm{~m}$ & $\begin{array}{l}\text { High serrated gamma ray value with } \\
\text { aggrading stacking patterns, this can } \\
\text { differentiated from each other by their } \\
\text { neutron and density log characters }\end{array}$ & $\begin{array}{l}\text { Offshore mudstone/ Lower } \\
\text { shoreface deposit }\end{array}$ \\
\hline
\end{tabular}

Figure 7 Gamma ray facies association from well log pattern used in defining depositional environments within the study area. 


\section{References}

Amajor, L. C. and Agbaire, D. W., 1989, Depositional History of reservoir sandstone, Akpor and Apara Oil Field Eastern Niger Delta. Nig. Jour. Petrol 12(14) p. 229-260

Asquith, G; Gibson, C; 1982, Basic well log Analysis for geologists AAPG methods in exploration series p. 175208

Bernard Biju-Duval and Edwin J. Swezey 2002, Sedimentary Geology p. 183 ISBN 2-7108-0802-1 Edition, Technip partial text.

Bradley D. Ritts 2004, Sedimentary records of Triassic intraplate extension in Northern China: Evidence from the Non-marine NW Ordos Basin Helan Shan. AAPG Bull p. 177 - 202.

Buatois, Luis; Mangano, M Gabriela, 2011 Ichnology: organism substrate interaction in space and time, New York Cambridge University press.

Casez, C. A., 2004. 'Overlap Zones, Growth Fault and Sedimentation: using High Resolution Gravity Data. Louisiana State University. Geological Society of America. Vol. 48 p.50

Celoria, Francis 1966 Delta as a Geographical concept in Greek Literature. The University of Chicago press books. P. 354-421

David T, 1995. Niger Delta Oil production, Reserves, Field Sizes Assessed. Industry Brief. Oil and Gas journal. P.120-127.

Egbu, O. C., G. C. Obi, C. O. Okugbe, A. W. Mode; 2009, Ichnofacies and Reservoir Property of offshore Deposition the Coastal Swamp Depobelt Niger Delta. P.19-38; 66-74

Ekweozor, C. M., and Okoye, N. V., 1980. Petroleum Source-bed Evaluation of Tertiary Niger Delta: AAPG Bulletin v.64, P $1251-1259$.

Environmental Resources Managers Ltd, 1997. Environmental Survey Final Report, phase 1 vol. 1: Environmental and socio-economic characteristics, Niger Delta Environmental Survey.

Etu - Efeotor, J. O, 2007 Principles of petroleum Geology Second edition. Paragraphics publishers Port Harcourt.

Frynas, J. G., 1999. Oil in Nigeria: Conflict and Litigation between Oil companies and Villages, Munster: Lit Verlag.

Garcia, R 1981 Depositional systems and their relation to gas accumulation in Sacramento valley, California. Amer. Assoc. Petrol. Geol. Bull. 65. P.48-59

Harald B, Wire Line depth determination, Rev 3.3, 2012. Available via society of professional well log analyst website: spwla.org. p.77-98

Hospers, J., 1965 'Gravity fields and structure of the Niger Delta Nigeria, West Africa' Bull. Geol. Amer., 76. P.201-297

Humphrey, G. S; Mitchell, P. B, 1983 A preliminary assessment of the role of bioturbation and rain-wash on sandstone hill slopes. P.95-179

Jardin, S. 1982: Geology and Tectonics of the South Atlantic Brazilian Basins. Geological society London special edition pp. $345-359$.

Joyjit D, Souvik S. 2007. Impact of Bioturbation on Reservoir Quality and Production - A Review. Journal of Geological society of India Vol 39 Issue 4. Pp 357 - 488.

Klieman, K. A., 2012. U.S. Oil companies, the Nigeria civil war and the Origin of Opacity in the Nigerian Oil Industry. Journal of American History. P.166-218 
Krumbein, W.C 1932. A history of the principle and methods of mechanical analysis. Jour. Sed. Petrol. Vol. 3 p. 59-184

La Croix; Andrew D.; Shahin E. 2015: A synthesis of depositional trends in intertidal and upper subtidal sediments across tidal-fluvial transition in the Fraser River. Canada Journal of sedimentary Research 85 6: 443 - 498.

Mascle, J., B. P. Bornhold and V. Renard 1973, Diapiric structures of the Niger Delta. Amer. Assoc. of Petrol. Geol. Bull. V57 p.96-138

Mascle, J., 1976, Submarine Niger Delta. Structural framework, Nig. Min. Geol. Soc. J v. 13(1). P.165-252

McBride, E. F; 1984 Compaction in sandstone - influence of reservoir quality AAPG Bulletin v.68. p.54-65

McLennan, J.; Stewart W., 2005. Deep water Africa reaches turning point, Oil and Gas Journal Vol. 103, 6; AB/inform global. P.34-52

Miall, A.D; 1984 Principles of sedimentary basin Analysis New York, Springer Verlag.

Michael, D. W. 2010 online publication: Reservoir quality assessment and prediction in clastic rocks. P.43-76

Nicholas, G, 2009 Principles of sedimentology and stratigraphy second edition. West Sussex.

Nsek, R. E., 2003, Hydrocarbon potential of reservoir sands in 'Anem Field, Niger. University of Port Harcourt. M.Sc. Thesis (unpublished).

Oti, M. N and Gibson, G. 1995 Geology of Deltas 315pp. Rotterdam, Brookfield. AAPG Edition.

Reyment, R. A. (1965) Aspects of the Geology of Nigeria. Ibadan University press, Ibadan Nigeria. P. 54-102

Ritter, M. E 2006. The physical environment: An Introduction to physical geography: The geologic work of streams. P.23-61

Schlumberger, 1989 Log Interpretation Principles and Application. 12-48

Selley, R. C. 2000 Applied sedimentology, Florida Academic press. P.54-70

Serra, O.; Abbott, H. T.; 1979. The Contribution of Logging data to Sedimentology and Stratigraphy. Society of Petroleum Engineers AIME Technical Conference. Dallas, Texas. P.17-52

Short, K. C. and Stauble, A. 1971 Outline Geology of Niger Delta. Bull. Amer. Assoc. Petrol. Geol., 51. P.50-87

Schlumberger Petrel software used: Petrel 2010 (student version).

Southard J. B. 2007, Sediment transport and sedimentary structures. Bull. Amer. Assoc. Petrol. Geol. P.66-79

Strahler, A and A. Strahler 2006: Introducing physical geography, Boston: Wiley \& sons. P98

Total E\&P Port Harcourt, 2016. Academic Data Set: Well Log, Mud Log, Cored data and other useful information.

Udden, J. A. 1914 Mechanical composition of clastic sediments - Geol. Soc. Amer. Bull. 25.

Wilson, M. D., Pittman, E. D 1977 Authigenic clay in sandstone - influence on reservoir properties and paleoenvironmental analysis. Journal of sedimentary petrology v. 47. P16-37

http://www.cell.com/trends/ecology.evolution.

google.com. 